\title{
Analysis of Large-Scale Dynamics and Gravity Waves under Shedding of Inactive Flow Components
}

\author{
ALmut GASSMANN \\ Leibniz Institute of Atmospheric Physics, Kühlungsborn, Germany
}

(Manuscript received 1 October 2018, in final form 25 April 2019)

\begin{abstract}
The Ertel's potential vorticity (EPV) budget equation does not see the contribution of an inactive EPV flux component $\nabla \theta \times \nabla B$ because it drops out when taking the divergence. A part of the actual EPV flux can always be interpreted as such an inactive component and is thus likewise shed from the EPV budget equation. The deviation from this inactive EPV flux is called the active EPV flux and the associated wind is called the active wind. The horizontal active wind is comparable to the ageostrophic wind. The vertical active wind component is similar to the isentropic displacement vertical wind. In contrast to the actual wind, the vertical active wind does not vanish at the surface, because the inactive wind blows along isentropes, which may intersect the ground. Transformed governing equations are derived as functions of the active wind components. The terms on the right of the transformed equations can be scrutinized with respect to their effects on the evolution of the atmospheric state. An idealized baroclinic wave in a dry atmosphere is discussed with focus on the fronts and the generation or depletion of kinetic energy. Since the vertical inactive wind does not necessarily vanish at the surface, the arising vertical active wind is responsible for the cooling (raising of isentropes) and the warming (sinking of isentropes) in the different regions of a cyclone. The new method allows for a unique separation of gravity waves and vortical modes. This facilitates the analysis of gravity wave generation and propagation from jets and fronts.
\end{abstract}

\section{Introduction}

Névir (2004) has been the first to provide the "most general stationary solution of the primitive equations" as

$$
\mathbf{v}_{\mathrm{st}}=\frac{\nabla \theta \times \nabla B}{\rho P}
$$

Here, $\theta$ is the potential temperature, $B=\mathbf{v}^{2} / 2+\Phi+c_{p} T$ is the Bernoulli function and $P=(2 \boldsymbol{\Omega}+\nabla \times \mathbf{v}) \cdot \nabla \theta / \rho$ is Ertel's potential vorticity (EPV). Previously, Schär (1993) discussed this solution rather in its form as an EPV flux $\rho \mathbf{v} P=\nabla \theta \times \nabla B$. He tried to interpret this flux as a statistically steady solution. The $\nabla \theta \times \nabla B$ flux vanishes when taking the divergence. Hence it does not have a contribution in the EPV budget equation. It is a "do nothing flux" (Haynes and McIntyre 1990). This fact lead to an inconclusive discussion among several authors (Davies-Jones 2003; Schär 2003; Bannon et al. 2003; Egger et al. 2015) whether this flux is of any

Corresponding author: Almut Gassmann, gassmann@iap-kborn.de relevance in the EPV budget equation and whether the EPV flux can be defined uniquely.

Any nonstationary EPV flux $\rho \mathbf{v} P$ can be cast as a $\nabla \theta \times \nabla B$ flux plus a deviation from it. In the present contribution, the $\nabla \theta \times \nabla B$ flux is called the inactive ${ }^{1}$ EPV flux and the associated wind is called the inactive part $\mathbf{v}_{\text {ia }}$ of the actual wind. This inactive wind can be interpreted as a more general brother of the geostrophic wind, as has been suggested by Lange (2002). The geostrophic wind arises from a scale analysis rather than the observation that the associated EPV flux is inactive in the EPV budget equation. Therefore, the comparison of both wind solutions seems a bit far-fetched. However, we shall see that the inactive horizontal wind components are very similar to the geostrophic wind components. The most important difference to the geostrophic wind is that the inactive wind has a significant vertical component and is nonlinear, because the kinetic energy and $P$ in the equation above depend on the wind field itself.

\footnotetext{
${ }^{1}$ The terminology "inactive" is here chosen to highlight that the flux is not seen in the EPV budget equation.
} 
Analogously to the ageostrophic wind, the active wind will here be defined as the deviation from the inactive wind. The governing equations may be transformed such that they depend merely on the active wind components, but not on the inactive wind components. Then it is of interest to inspect which properties of the active flow determine the evolution of the three main measures of atmospheric hydro-thermodynamics: vorticity, horizontal divergence and potential temperature. Whereas the differentiation into active and inactive wind components has already been introduced in Gassmann (2014), the role of the active wind in the transformed governing equations has not yet been scrutinized.

How do large-scale vorticity dynamics and gravity wave dynamics can be interpreted under the shedding of inactive flow components? On the one hand, the active motions are responsible for the baroclinic development including the generation of kinetic energy and the poleward heat flux. The vorticity equation and the thermodynamic equation are decisive for this development in a baroclinic atmosphere. On the other hand, the equation for horizontal divergence is decisive for the evolution of gravity waves that form along fronts and jets (Plougonven and Zhang 2014). Especially the latter topic is of interest, because the gravity wave life cycle including gravity wave generation, propagation and breaking is essential for the understanding of the general dynamics of the atmosphere. Even though gravity waves are only of local and minor importance in the troposphere, they determine largely the circulation in the middle atmosphere (Andrews et al. 1987). When diagnosing the spontaneously generated gravity waves from jets and fronts [e.g., O'Sullivan and Dunkerton (1995) or Plougonven and Snyder (2007)], often the horizontal divergence is inspected, but this measure may have an own balanced part, which masks the proper gravity waves. Therefore, Mirzaei et al. (2017) tested several balance relations in order to remove this effect. The present work presents an alternative way for diagnosing gravity waves, because the evolution of the horizontal divergence can be related to some properties of the active wind.

Vertical motions are usually disentangled into a vanishing geostrophic and a nonvanishing ageostrophic portion. Traditionally, the vertical wind is seen as a secondary ageostrophic motion, and the diagnostic omega equation is decisive for its determination within the quasigeostrophic theory (Phillips 1963). In contrast, the inactive flow has a vertical wind component guaranteeing that the wind blows along sloped isentropes. That means that the thermodynamic field remains unchanged if the flow is found to be inactive. The vertical active wind is responsible for the upward and downward motions of isentropes, which leads to cooling or warming, respectively. Making those active vertical motions visible may sharpen our picture of baroclinic motions, as has been brought forward by Papritz and Spengler (2015) who studied the tendencies of isentropic slopes.

The present contribution aims at shedding light on the sketched different aspects of the active flow components. As already mentioned, the governing atmospheric equations are first reformulated (section 2) such that they only depend on the active wind components and the inactive part cancels out there. Via deriving the vorticity and divergence equations that depend on the active wind it is possible to disentangle the driving for vortical modes and gravity wave dynamics. The vertical active wind is found essential for the development of the thermodynamic structure of the atmosphere. Exemplarily, idealized baroclinic waves in a dry atmosphere are inspected and discussed in section 3. Section 4 concludes the paper.

\section{Transformation of the governing equations}

\section{a. Transformation without reference to a specific vertical coordinate system}

The governing equations for the dry atmosphere read in Eulerian form as

$$
\begin{aligned}
& \partial_{t} \mathbf{v}=-\boldsymbol{\omega}_{a} \times \mathbf{v}-\nabla B+c_{p} \Pi \nabla \theta+\mathbf{F}_{r}, \\
& \partial_{t} \theta=-\mathbf{v} \cdot \nabla \theta+Q, \\
& \partial_{t} \rho=-\nabla \cdot(\rho \mathbf{v}) .
\end{aligned}
$$

Here the formulation of the velocity equation in (1) is intentionally given in vector invariant form. The generalized Coriolis term uses the absolute vorticity vector $\boldsymbol{\omega}_{a}=2 \boldsymbol{\Omega}+\nabla \times \mathbf{v}$ and the gradient terms are given as the gradient of the Bernoulli function $B=K+\Phi+c_{p} \Pi \theta$ and a term proportional to the gradient of the potential temperature $\theta$. Specifically, $\mathbf{v}$ is the velocity, $\boldsymbol{\Omega}$ is Earth's angular velocity, $\Pi=\left(p / p_{\text {ref }}\right)^{R / c_{p}}$ is the Exner pressure, $\rho$ is the density, $K=\mathbf{v}^{2} / 2$ is the specific kinetic energy, and $\Phi$ is the geopotential.

Searching for a stationary $\left(\partial_{t}=0\right)$ solution to these equations without friction $\left(\mathbf{F}_{r}=0\right)$ and diabatic heating $(Q=0)$ gives

$$
\begin{aligned}
\mathbf{v}_{\mathrm{ia}} & =(\nabla \theta \times \nabla B) /(\rho P), \\
\Pi_{\mathrm{ia}} & =\left(\boldsymbol{\omega}_{a} \cdot \nabla B\right) /\left(c_{p} \rho P\right), \\
\mathrm{DSI}^{*} & =-\nabla \cdot\left(\rho \mathbf{v}_{\mathrm{ia}}\right)=0,
\end{aligned}
$$


as already noted by Lange $(2002)^{2}$, Névir (2004), Weber and Névir (2008), and Gassmann (2014). In (6), DSI* is the dynamical state index introduced by Weber and Névir (2008). They and subsequent authors (Müller et al. 2018) used this index in the form $-\nabla \cdot\left(\rho \mathbf{v}_{\text {ia }}\right)=\rho \mathbf{v}_{\text {ia }} \cdot \nabla \ln P$, which inspects the dynamical activity in the atmosphere via the EPV evolution. The index ia in the above expressions should be read as "inactive" as has been motivated in the introduction.

The inactive wind (4) blows along intersection lines of $B$ with isentropic surfaces. It deviates from the well-known geostrophic wind solution in its three-dimensionality, hence it contains also a vertical component, and it is nonlinear, hence it considers the gradient of the kinetic energy as an additional force and contains in the denominator the absolute vorticity instead of the Coriolis parameter $f$.

When assuming the hydrostatic approximation, the components of the inactive wind in the Cartesian coordinate system yield

$$
\begin{aligned}
& u_{\mathrm{ia}}=\left(-c_{p} \theta \partial_{y} \Pi-\partial_{y} K+\frac{\partial_{y} \theta}{\partial_{z} \theta} \partial_{z} K\right) / \zeta_{a}^{*}, \\
& v_{\mathrm{ia}}=\left(c_{p} \theta \partial_{x} \Pi+\partial_{x} K-\frac{\partial_{x} \theta}{\partial_{z} \theta} \partial_{z} K\right) / \zeta_{a}^{*}, \\
& w_{\mathrm{ia}}=\left[\frac{\partial_{x} \theta}{\partial_{z} \theta}\left(c_{p} \theta \partial_{y} \Pi+\partial_{y} K\right)-\frac{\partial_{y} \theta}{\partial_{z} \theta}\left(c_{p} \theta \partial_{x} \Pi+\partial_{x} K\right)\right] / \zeta_{a}^{*},
\end{aligned}
$$

where

$$
\zeta_{a}^{*}=\rho P / \partial_{z} \theta=f+\omega_{z}+\frac{\partial_{x} \theta}{\partial_{z} \theta} \omega_{x}+\frac{\partial_{y} \theta}{\partial_{z} \theta} \omega_{y}
$$

is an abbreviation for the absolute vorticity component perpendicular to isentropic surfaces. The leading terms in the numerator and denominator of the horizontal inactive wind components (7) and (8) reveal the well-known geostrophic wind. The other terms refer to the nonlinearity of the inactive wind. The vertical inactive wind (9) signifies baroclinity, because a dominating part of the numerator measures the intersection of $\Pi$ and $\theta$ surfaces on the same height $\mathbf{k} \cdot\left(\nabla \theta \times c_{p} \theta \nabla \Pi\right)$. Papritz and Spengler (2015) noted that the magnitude of the slope of the isentropes $\left|\nabla_{h} \theta / \partial_{z} \theta\right|=\left|\left(\partial_{x} \theta / \partial_{z} \theta \mathbf{i}\right)+\left(\partial_{y} \theta / \partial_{z} \theta \mathbf{j}\right)\right|$-which also occurs in (9)-resembles the expression for the growth rate of the most unstable mode in the Eady model. Like

\footnotetext{
${ }^{2}$ Lange mentioned in that textbook that this solution has been actually found by Peter Névir, but had not yet been published at that time.
}

Papritz and Spengler (2015), it is noted that the existence of this slope does not immediately lead to the release of the baroclinic instability. Whereas they decided investigating the tendency of the slope of an isentrope, here the depletion of the baroclinity will be characterized by the deviation of the actual vertical wind $w$ from the inactive vertical wind $w_{\text {ia }}$.

The vertical inactive wind (9) may also be expressed as the advection of $\theta$ with the inactive horizontal wind divided by $\partial_{z} \theta$ :

$$
w_{\text {ia }}=-u_{\text {ia }} \partial_{x} \theta / \partial_{z} \theta-v_{\text {ia }} \partial_{y} \theta / \partial_{z} \theta .
$$

This vertical inactive wind is the isentropic upglide in case of a stationary state.

It is not mandatory that the flow is three-dimensional for the definition of the inactive wind. Here we only focus on this three-dimensional case. Appendix B describes how a stationary flow in the vertical slice can be characterized as being inactive.

Clearly, such a stationary solution cannot describe a global hypothetical flow field in the real atmosphere, because an impermeable boundary such as the surface is just ignored by this stationary solution. The atmospheric flow cannot become truly stationary unless $w_{\text {ia }}$ vanishes at the flat surface. As mentioned, $w_{\text {ia }}$ becomes significant in a baroclinic atmosphere. Then, the Bernoulli function isolines on isentropes may intersect the ground and a purely inactive motion along isentropes may become impossible. We must conclude that only a barotropic flow may become truly stationary under adiabatic conditions. Consequently, the deviations from the inactive wind are most important for any evolving atmospheric dynamics.

The deviation of the actual wind is now defined as the active wind $\mathbf{v}_{a}=\mathbf{v}-\mathbf{v}_{\text {ia }}$. When computing the inactive wind with (4) for such a nonstationary flow field, the relative vorticity and the kinetic energy therein are defined with the actual wind $\mathbf{v}$, not with the inactive wind $\mathbf{v}_{\text {ia }}$. Now, the equations of motion can be transformed such that forces that cancel out each other are made invisible and only net forces reside in the Eulerian frame. The according steps toward the local transformed momentum equation are given in the following:

$$
\begin{aligned}
\partial_{t} \mathbf{v} & =-\boldsymbol{\omega}_{a} \times\left(\mathbf{v}_{a}+\mathbf{v}_{\mathrm{ia}}\right)-\nabla B+c_{p} \Pi \nabla \theta+\mathbf{F}_{r} \\
& =-\boldsymbol{\omega}_{a} \times \mathbf{v}_{a}-\boldsymbol{\omega}_{a} \times \frac{\nabla \theta \times \nabla B}{\rho P}-\nabla B+c_{p} \Pi \nabla \theta+\mathbf{F}_{r} \\
& =-\boldsymbol{\omega}_{a} \times \mathbf{v}_{a}-\frac{\left(\boldsymbol{\omega}_{a} \cdot \nabla B\right) \nabla \theta-\rho P \nabla B}{\rho P}-\nabla B+c_{p} \Pi \nabla \theta+\mathbf{F}_{r} \\
& =-\boldsymbol{\omega}_{a} \times \mathbf{v}_{a}+c_{p}\left(\Pi-\frac{\boldsymbol{\omega}_{a} \cdot \nabla B}{c_{p} \rho P}\right) \nabla \theta+\mathbf{F}_{r},
\end{aligned}
$$




$$
\partial_{t} \mathbf{v}=-\boldsymbol{\omega}_{a} \times \mathbf{v}_{a}+c_{p} \Pi_{a} \nabla \theta+\mathbf{F}_{r},
$$

where $\Pi_{a}=\Pi-\Pi_{\mathrm{ia}}$ is the active Exner pressure. The term with the active Exner pressure becomes only significant in the vertical velocity equation whereas it is always small in the horizontal velocity equations. This term vanishes if horizontal motions on an isentropic surface are considered. In this case only the first term is left besides the friction term.

The thermodynamic equation can also be transformed such as to depend on the active wind vector

$$
\partial_{t} \theta=-\left(\mathbf{v}_{\mathrm{ia}}+\mathbf{v}_{a}\right) \cdot \nabla \theta+Q=-\mathbf{v}_{a} \cdot \nabla \theta+Q .
$$

This transformation indicates that the potential temperature is effectively transported by the active wind. Any advection with the inactive wind does not have any effect on the evolution of the potential temperature field. Here the advantage of a three-dimensional inactive wind becomes most obvious. Knowing that the active horizontal wind-just like the classical ageostrophic wind-does not contribute much to the horizontal advection of $\theta$ because it is much smaller than the inactive contribution, the advection with the vertical active wind dominates the advection term in (14). The true transport of $\theta$ with the inactive horizontal components is transferred to the advection with the vertical active wind, because $w_{a}=w-w_{\text {ia }}=w-u_{\text {ia }} \partial_{x} \theta-v_{\text {ia }} \partial_{y} \theta$. The inactive horizontal wind is predominantly parallel to the isobars and leads to a horizontal $\theta$ transport if isentropes and isobars cross each other at the same height, as is the case in a baroclinic environment.

From (13) and (14) it is straightforward to deduce the EPV budget equation. First, the momentum equation in (13) is crossed with $-\nabla \theta$ :

$$
\begin{aligned}
& -\nabla \theta \times \partial_{t} \mathbf{v}=\nabla \theta \times\left(\boldsymbol{\omega}_{a} \times \mathbf{v}_{a}\right)-\nabla \theta \times \mathbf{F}_{r} \\
& -\nabla \theta \times \partial_{t} \mathbf{v}=-\mathbf{v}_{a}\left(\nabla \theta \cdot \boldsymbol{\omega}_{a}\right)+\boldsymbol{\omega}_{a}\left(\mathbf{v}_{a} \cdot \nabla \theta\right)-\nabla \theta \times \mathbf{F}_{r} .
\end{aligned}
$$

Now, the thermodynamic equation in (14) is used to replace $\mathbf{v}_{a} \cdot \nabla \theta$ and all time derivatives are collected on the left-hand side:

$$
-\nabla \theta \times \partial_{t} \mathbf{v}+\boldsymbol{\omega}_{a} \partial_{t} \theta=-\rho \mathbf{v}_{a} P+\boldsymbol{\omega}_{a} Q-\nabla \theta \times \mathbf{F}_{r} .
$$

The right-hand side of this equation is the full EPV flux. The operation $-\nabla \theta \times$ above had guaranteed that this EPV flux does not cross isentropic surfaces. Taking the divergence of (16) leads directly to the EPV budget equation:

$$
\partial_{t}(\rho P)=-\nabla \cdot\left(\rho \mathbf{v}_{a} P-\boldsymbol{\omega}_{a} Q+\nabla \theta \times \mathbf{F}_{r}\right) .
$$

The full EPV flux on the right-hand side of (16) contains the inactive flux $\rho \mathbf{v}_{\mathrm{ia}} P=\nabla \theta \times \nabla B$, because $\rho \mathbf{v}_{a} P=\rho \mathbf{v} P-\rho \mathbf{v}_{\mathrm{ia}} P$. The divergence of this inactive EPV flux vanishes, $\nabla \cdot(\nabla \theta \times \nabla B)=0$. This unique character of the inactive EPV flux was highlighted in the introduction and taken as a motivation for the steps done so far. The fact that the inactive EPV flux vanishes under taking the divergence does not mean that this term does not play a role in the flux itself. The dynamics generates a certain unique EPV flux on the right-hand side of (16), only the application of the divergence operator in (17) leads to the often discussed ambiguities.

Completing the set of transformed governing equations, the continuity equation reads

$$
\begin{aligned}
& \partial_{t} \rho=-\nabla \cdot\left(\rho \mathbf{v}_{a}+\rho \mathbf{v}_{\text {ia }}\right) \\
& \partial_{t} \rho=-\nabla \cdot\left(\rho \mathbf{v}_{a}\right)+\mathrm{DSI}^{*},
\end{aligned}
$$

where DSI* $=-\nabla \cdot\left(\rho \mathbf{v}_{\text {ia }}\right)$ is again the dynamic state index introduced by Weber and Névir (2008). The DSI* appears as a pretended source term for mass when interpreting the continuity equation from the perspective of the mass that is "actively" transported around. The mass that is "inactively" transported around is represented by the DSI*.

The newly introduced transformed equations in (13), (14), and (18) make inactive dynamics invisible. Therefore, they are closely related to the transformed Eulerian mean (TEM) equations (Andrews and McIntyre, 1976) when averaged. Likewise, the aim when developing the TEM equations was to exclude the signature of ideal noninteracting waves from the diagnosis of mean atmospheric behavior (Boyd 1976; Andrews et al. 1987). Gassmann (2014) discussed the similarities and differences between the residual winds and the zonally averaged active winds. The main assumption of the TEM framework, namely that the residual mass flux is divergence free in the meridional plane and allows for a meridional streamfunction to be specified, is not reproduced by the transformed continuity equation in (18), even if this equation is averaged. The DSI* is a source term in the transformed continuity equation and highlights that the mass flux may change its meaning between belonging to the inactive flow part and the active flow part. This cannot happen in the TEM framework, where the respective circulations in the Stokes drift and the actual waves remain separated by definition.

Last, let us inspect the energy conversions of kinetic energy $K$. They can also be interpreted as being associated with the active wind: ${ }^{3}$

\footnotetext{
${ }^{3}$ Note here the vector algebra rule $\mathbf{A} \cdot(\mathbf{A} \times \mathbf{B})=0$ in the forms $(\nabla \theta \times \nabla B) \cdot \nabla B /(\rho P)=0$ and $(\nabla \theta \times \nabla B) \cdot \nabla \theta /(\rho P)=0$ and the definition of $B=c_{p} \Pi \theta+K+\Phi$.
} 


$$
\begin{aligned}
\partial_{t} K= & -\mathbf{v} \cdot\left(\boldsymbol{\omega}_{a} \times \mathbf{v}\right)-\left(\mathbf{v}_{\mathrm{ia}}+\mathbf{v}_{a}\right) \cdot \nabla B \\
& +c_{p} \Pi\left(\mathbf{v}_{\mathrm{ia}}+\mathbf{v}_{a}\right) \cdot \nabla \theta+\mathbf{v} \cdot \mathbf{F}_{r} \\
\partial_{t} K= & -\mathbf{v}_{a} \cdot \nabla B+c_{p} \Pi \mathbf{v}_{a} \cdot \nabla \theta+\mathbf{v} \cdot \mathbf{F}_{r} \\
\partial_{t} K= & -\mathbf{v}_{a} \cdot \nabla K-\mathbf{v}_{a} \cdot \nabla \Phi-\mathbf{v}_{a} c_{p} \theta \cdot \nabla \Pi+\mathbf{v} \cdot \mathbf{F}_{r} \\
\partial_{t} K \approx & -\mathbf{v}_{a, h} c_{p} \theta \cdot \nabla_{h} \Pi-\mathbf{v}_{a, h} \cdot \nabla_{h} K-w_{a} \partial_{z} K+\mathbf{v} \cdot \mathbf{F}_{r} .
\end{aligned}
$$

The last equation results from assuming the hydrostatic balance. This result does not mean that for instance adiabatic conversion - the work of the pressure gradient term - is only accomplished by the active wind part. The inactive wind has also a contribution to the adiabatic conversion term, but this effect is exactly offset by the advection of the kinetic energy with the inactive wind. Hence, we can only say that after disregarding such cancellations, the pressure gradient term dotted with the active wind represents the apparent adiabatic conversion term.

\section{b. Transformed vertical vorticity and horizontal divergence equations in the $z$-coordinate system}

In the $z$-coordinate system, the cumbrous $\Pi_{a}$ term in the horizontal velocity equation in (13) can be eliminated by inserting the vertical velocity equation, which yields

$$
\begin{aligned}
\partial_{t} \mathbf{v}_{h}-\frac{\nabla_{h} \theta}{\partial_{z} \theta} \partial_{t} w & =-\mathbf{k} \times \mathbf{v}_{a, h} \zeta_{a}^{*}+\mathbf{k} \times w^{*} \boldsymbol{\omega}_{h}+\mathbf{F}_{r, h}-\frac{\nabla_{h} \theta}{\partial_{z} \theta} F_{r, z} \\
& =-\mathbf{k} \times\left(\mathbf{g}_{1}+\mathbf{g}_{2}\right)+\mathbf{F}_{r, h}-\frac{\nabla_{h} \theta}{\partial_{z} \theta} F_{r, z} .
\end{aligned}
$$

This is in fact the prognostic equation for that part of the wind (expressed with $z$ coordinates) that blows along an isentrope ${ }^{4}$. The isentropic slope $\nabla_{h} \theta / \partial_{z} \theta$ multiplied with $\partial_{t} w$ or $F_{r, z}$ is negligible in (20). It only becomes important for vigorously nonhydrostatic motions, as for instance small-scale breaking gravity waves. The vertical velocity

$$
w^{*}=\left(\mathbf{v}_{a} \cdot \nabla \theta\right) / \partial_{z} \theta
$$

is the measured vertical motion of an isentrope. Papritz and Spengler (2015) and Hoskins et al. (2003) call this net advective vertical wind the isentropic displacement vertical wind. With this vertical wind, the potential temperature equation reads simply as

$$
\partial_{t} \theta=-w^{*} \partial_{z} \theta+Q
$$

\footnotetext{
${ }^{4}$ This means that (20) may also be obtained by taking $\mathbf{k} \times(15)$.
}

The other newly defined abbreviations in (20) are

$$
\begin{aligned}
& \mathbf{g}_{1}=\mathbf{v}_{a, h} \zeta_{a}^{*} \\
& \mathbf{g}_{2}=-w^{*} \boldsymbol{\omega}_{h} .
\end{aligned}
$$

One might fear that the horizontal vector $\mathbf{g}_{1}$ becomes undefined when the absolute vorticity $\zeta_{a}^{*}$ vanishes in equatorial regions. This is, however, not the case if considering the full form with the actual wind and the inactive wind as in (7) and (8):

$$
\mathbf{g}_{1}=\left(\mathbf{v}_{h}-\mathbf{v}_{\mathrm{ia}, h}\right) \zeta_{a}^{*}=\mathbf{v}_{h} \zeta_{a}^{*}-\mathbf{k} \times\left(c_{p} \theta \nabla \Pi+\nabla K-\nabla \theta \frac{\partial_{z} K}{\partial_{z} \theta}\right) .
$$

Hence, for a vanishing absolute vorticity $\zeta_{a}^{*}$ the vector $\mathbf{g}_{1}$ selects the horizontal processes like the pressure gradient term and the horizontal advection. The vector $\mathbf{g}_{2}$ depends on the horizontal vorticity vector $\boldsymbol{\omega}_{h}$ and therefore selects the net vertical advection.

When aiming at disentangling vortical dynamics from gravity wave dynamics, transfomed equations are required for vertical vorticity $\zeta=\omega_{z}$ and horizontal divergence $\delta$. They are obtained as

$$
\begin{aligned}
\partial_{t} \zeta= & -\nabla_{h} \cdot\left(\mathbf{g}_{1}+\mathbf{g}_{2}\right)+\mathbf{k} \cdot \nabla \times \mathbf{F}_{r, h} \\
& +\mathbf{k} \cdot \nabla \times\left[\frac{\nabla_{h} \theta}{\partial_{z} \theta}\left(\partial_{t} w-F_{r, z}\right)\right]
\end{aligned}
$$

and

$$
\begin{aligned}
\partial_{t} \delta= & \mathbf{k} \cdot \nabla \times\left(\mathbf{g}_{1}+\mathbf{g}_{2}\right)+\nabla_{h} \cdot \mathbf{F}_{r, h} \\
& +\nabla_{h} \cdot\left[\frac{\nabla_{h} \theta}{\partial_{z} \theta}\left(\partial_{t} w-F_{r, z}\right)\right] .
\end{aligned}
$$

In all the following discussions it is assumed that $\mathbf{F}_{r}$ and $\partial_{t} w \nabla_{h} \theta / \partial_{z} \theta$ are small compared to the other terms. Then, only the vectors $\mathbf{g}_{1}$ and $\mathbf{g}_{2}$ determine the evolution of the flow field. It is seen from (26) that those vectors appear as active vorticity flux vectors. Their negative divergences determine the evolution of the vorticity field. Note that the vorticity equation does not explicitly possess a baroclinity (solenoidal) term. The remaining twisting and vertical advection terms sum to the $\mathbf{g}_{2}$ term.

The divergence equation in (27) takes an even more interesting form than the vorticity equation. First, the rotational aspects of the same two active vorticity flux vectors $\mathbf{g}_{1}$ and $\mathbf{g}_{2}$ determine the evolution of the divergence. Consequently, gravity waves may be filtered out from the equations by suppressing the rotational part of the active vorticity flux vectors. Second, a lot of known 
terms do not occur explicitly. These are those that turn out to be very close to a classical nonlinear balance equation (NBE). Namely, from

$$
0=-\boldsymbol{\omega}_{a} \times \mathbf{v}_{\mathrm{ia}}-\nabla B+c_{p} \Pi_{\mathrm{ia}} \nabla \theta
$$

follows trivially

$$
0=\nabla_{h} \cdot\left(-\boldsymbol{\omega}_{a} \times \mathbf{v}_{\mathrm{ia}}-\nabla B+c_{p} \Pi_{\mathrm{ia}} \nabla \theta\right),
$$

which gives under the hydrostatic assumption and with defining the vertical vorticity with inactive horizontal wind components as $\zeta_{\mathrm{ia}}$ :

$$
\begin{aligned}
0= & f \zeta_{\mathrm{ia}}-\left.\nabla_{h}^{2} \Phi\right|_{p}-u_{\mathrm{ia}} \partial_{y} f+\zeta \zeta_{\mathrm{ia}}+v_{\mathrm{ia}} \partial_{x} \zeta-u_{\mathrm{ia}} \partial_{y} \zeta \\
& -\nabla_{h}^{2} K+\mathbf{k} \cdot\left(\boldsymbol{\omega}_{h} \times \nabla_{h} w_{\mathrm{ia}}\right)+w_{\mathrm{ia}} \nabla_{h}^{2} w-w_{\mathrm{ia}} \partial_{z} \delta \\
& -c_{p} \nabla_{h} \cdot\left(\Pi_{a} \nabla_{h} \theta\right),
\end{aligned}
$$

where $\left.\right|_{p}$ refers to an operation along pressure surfaces. The first two terms establish the most simple and well known geostrophic balance, the third term is the $\beta$ term and the fourth to seventh terms correspond to the wellknown Jacobi terms in the traditional nonlinear balance equation [see, e.g., Raymond and Jiang (1990), their Eq. (13)], if the velocities and vorticities are expressed with a geostrophic streamfunction. Most of the other terms result from three-dimensionality. The right-hand side of the prognostic divergence equation in (27) selects thus only gravity wave dynamics and the terms describing approximately the classical nonlinear balance in (30) are separated out beforehand.

Equation (30) is not yet a filter condition for gravity waves, rather it is another form of the definition of the inactive flow, because it has been derived from (28). A filtering of gravity waves from the flow field is achieved by suppressing the forcing to the divergence equation in (27) completely.

Formally, it is possible to separate the vector $\mathbf{g}=\mathbf{g}_{1}+$ $\mathbf{g}_{2}$ into a vortical and a gravity wave part $\mathbf{g}=\mathbf{g}^{\mathrm{VM}}+$ $\mathbf{g}^{\mathrm{GW}}=\nabla_{h} \chi+\mathbf{k} \times \nabla \psi$, where the part with the velocity potential $\chi$ is responsible for the vortical part and the streamfunction $\psi$ part describes the gravity wave modes. Allowing only for the velocity potential part of $\mathbf{g}=$ $\mathbf{g}^{\mathrm{VM}}=\nabla_{h} \chi$ in a prognostic velocity equation like (20) would filter out gravity waves from the flow.

\section{Baroclinic wave development}

Idealized simulations of baroclinic developments are carried out with the ICON-IAP model (Gassmann 2013, 2018). The ICON-IAP model is a global nonhydrostatic model that uses a hexagonal C-grid mesh. Only recently (Gassmann 2018) the numerics could be improved such that nonlinear momentum advection corresponds to the classical energy conserving C-grid scheme on quadrilaterals (Sadourny 1975). The model is run in a dry mode. As soon as strong shears at fronts are created by the dynamics, they have to be smoothed by diffusion. Therefore, horizontal momentum diffusion and the associated frictional heating are taken into account from the physics parameterization package. The model is run for 12 days and with 70 vertical layers up to $35-\mathrm{km}$ height and a horizontal grid spacing of about $30 \mathrm{~km}$. The number of main grid boxes in the model is $10 \times$ $2^{2} \times 4^{7}+2=655362$. The initial conditions for the baroclinic wave are given in appendix A. The wave is triggered by a cold air plume in the lower troposphere.

\section{a. The inactive wind on the 290-K isentrope}

Figure 1 displays the height of the $290-\mathrm{K}$ isentrope and the isolines of the Bernoulli function for the initial conditions and at day 10. According to (4), the inactive wind blows in direction along the intersection lines of $B$ and $\theta$. Initially (top panel), the $B$ isolines are parallel to the height on the isentropic surface. The inactive wind and so the inactive EPV flux are purely horizontal. At day 10, the inactive horizontal wind blowing along the isolines of $B$ is very variable in speed (middle panel). $A$ jet with enhanced wind is seen between $45^{\circ}-60^{\circ} \mathrm{N}$ and $160^{\circ}-165^{\circ} \mathrm{E}$ and also between $45^{\circ}-50^{\circ} \mathrm{N}$ and west of $135^{\circ} \mathrm{E}$. The $B$ isolines cross the height isolines. This comes along with the respective vertical inactive wind as displayed in the bottom panel. The $B$ isolines even intersect the surface. Therefore the inactive vertical wind and thus the vertical EPV flux may cross the surface. At the surface, the vertical active wind must have the opposite sign than the vertical inactive wind because $w_{s}=$ $0=w_{s, \text { ia }}+w_{s, a}$. Therefore, the isentropic surface must be raised and swept over the surface in the cold sector of the cyclone. On the other hand, the isentrope is pushed down in the warm sector.

\section{b. Lower-tropospheric structures}

The panels of Fig. 2 compare the winds and the active winds for a train of cyclones at a level in the lower troposphere. It is striking that the order of magnitude of $w_{a}$ can become much larger than the order of magnitude of $w$. The vertical wind has a maximum in the frontal areas, whereas $w_{a}$ vanishes at the fronts. That means that the front itself is very locally in an inactive state, hence $w=w_{\text {ia }}$. The first cyclone is still growing and features positive $w_{a}$ in the cold sector and negative $w_{a}$ in the warm sector. The southern part of the frontal area in the second cyclone does not show much activity. The active 

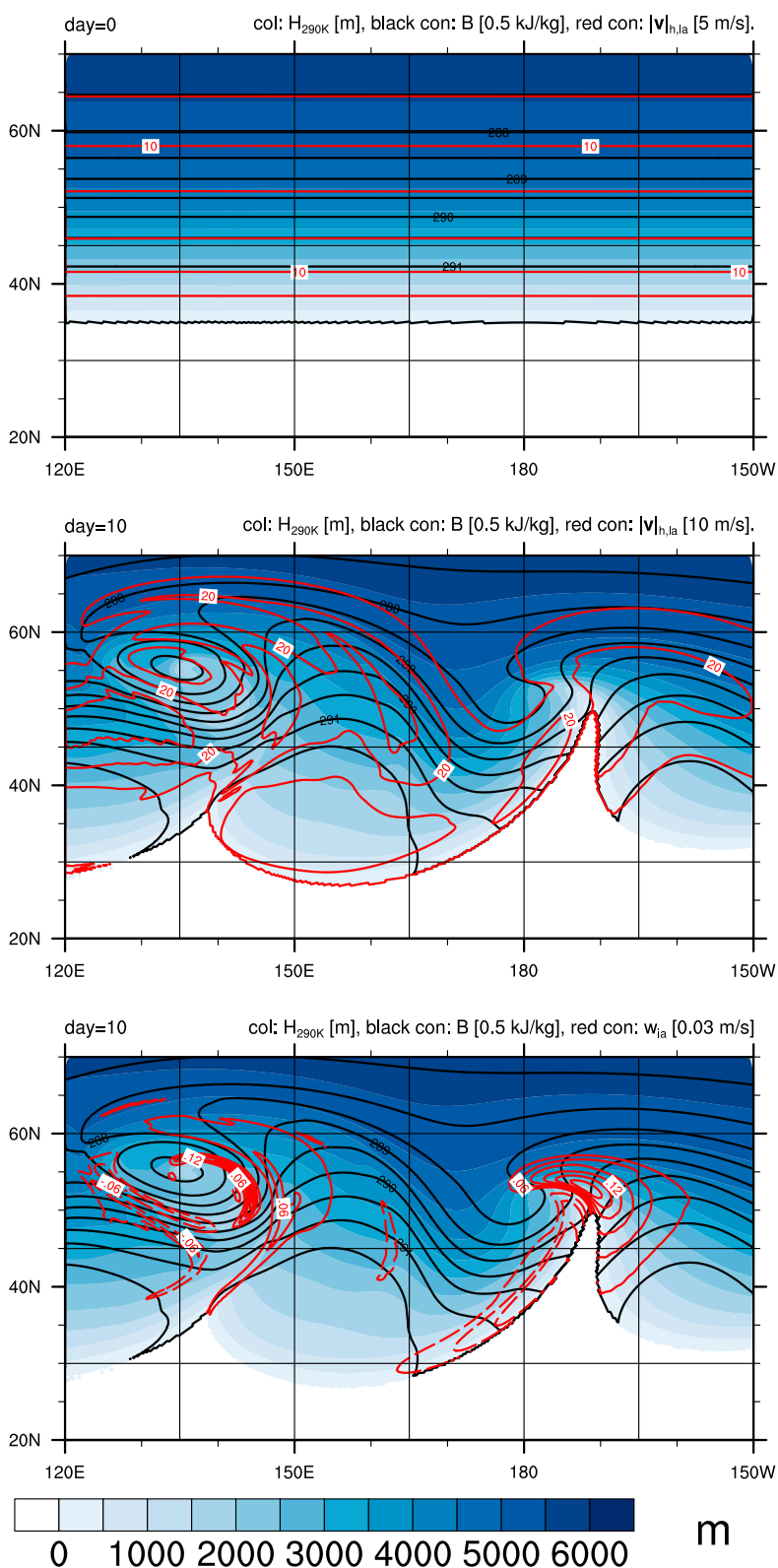

$\mathrm{m}$

FIG. 1. Height of the $290-\mathrm{K}$ isentrope together with $B$ (black isolines) and (top),(middle) $\left|\mathbf{v}_{h, \text { ia }}\right|$ (red isolines) or (bottom) $w_{\text {ia }}$ (red isolines) at (top) day 0 and (middle),(bottom) day 10.

vertical wind $w_{a}$ is already small even though a considerable vertical wind $w$ is present. Consequently, this frontal development has matured and the front is in equilibrium.

Figure 3 left displays the isentropic displacement vertical wind $w^{*}$ instead of $w_{a}$. The pattern of both vertical winds are comparable, but $w^{*}$ is less intense. This means that the horizontal advection with the active wind counteracts the vertical advection with $w_{a}$ to some degree. This can be called the isentropic upglide contribution of the active wind. The overall signatures show rising motions and thus adiabatic cooling in the cold sector. Sinking motion in the warm sector of the cyclone is associated with adiabatic warming. The downward/ upward motion with $w$ in the cold/warm sector that would lead to an adiabatic warming/cooling is more than compensated by horizontal advection of cold/ warm air with $\mathbf{v}_{h}$.

The horizontal active wind is not predominantly responsible for heat transport, but for the evolution of the dynamical field. As can be seen in Fig. 2, the horizontal active wind vectors have a considerably distinct direction compared to the actual wind vectors. This is expected from the analogy between the horizontal active and ageostrophic winds. Figure 3 right displays the active horizontal wind vectors together with the kinetic energy and the Exner pressure. This allows for a qualitative estimation of the evolution of the kinetic energy according to the first two terms on the right of (19). We see that kinetic energy is generated whenever the vectors have a component pointing toward the low pressure center. As expected, this is always true for approaching and deepening cyclones. There, the active wind is very similar to the classical isallobaric wind. The active wind may also have a component being opposite to that of the pressure gradient, as can be seen to the southwest of the second cyclone. Kinetic energy is only created at the inner side of the jet via the pressure gradient term, whereas it is destroyed at the outer flank. The more interesting effect is that kinetic energy is transported away from the jet core with $\mathbf{v}_{a}, h$ toward both lateral directions. It can be estimated that this effect is similar on both sides, because the gradient of the kinetic energy is larger at the inner flank, where the magnitude of $\mathbf{v}_{a, h}$ is small, whereas the opposite is true for the outer flank. The active vertical advection of kinetic energy transports likewise kinetic energy away from the jet core as can be inferred from Fig. 5 at a longitude of about $130^{\circ} \mathrm{E}$.

The vectors $\mathbf{g}_{1}$ and $\mathbf{g}_{2}$ represent the forcing terms to the horizontal momentum equation in (20). Figure 4 displays them separately. The direction of $\mathbf{g}_{1}$ is the same as for $\mathbf{v}_{a, h}$, but the magnitude differs because of the weighting with $\zeta_{c}^{*}$ This weighting alters its meaning toward a net vorticity flux term. The vector $-\mathbf{k} \times \mathbf{g}_{2}$ represents the active vertical advection of momentum. In large areas, like the evolving cold air region of the fist cyclone and the areas to the northeast of both cyclones, the vectors $\mathbf{g}_{1}$ and $\mathbf{g}_{2}$ oppose each other in direction. This means that the kinetic energy that is generated by the horizontal forces is at least partly transported away by the vertical advection of momentum with $w^{*}$. It is interesting that $\mathbf{g}_{1}$ and $\mathbf{g}_{2}$ point in the 

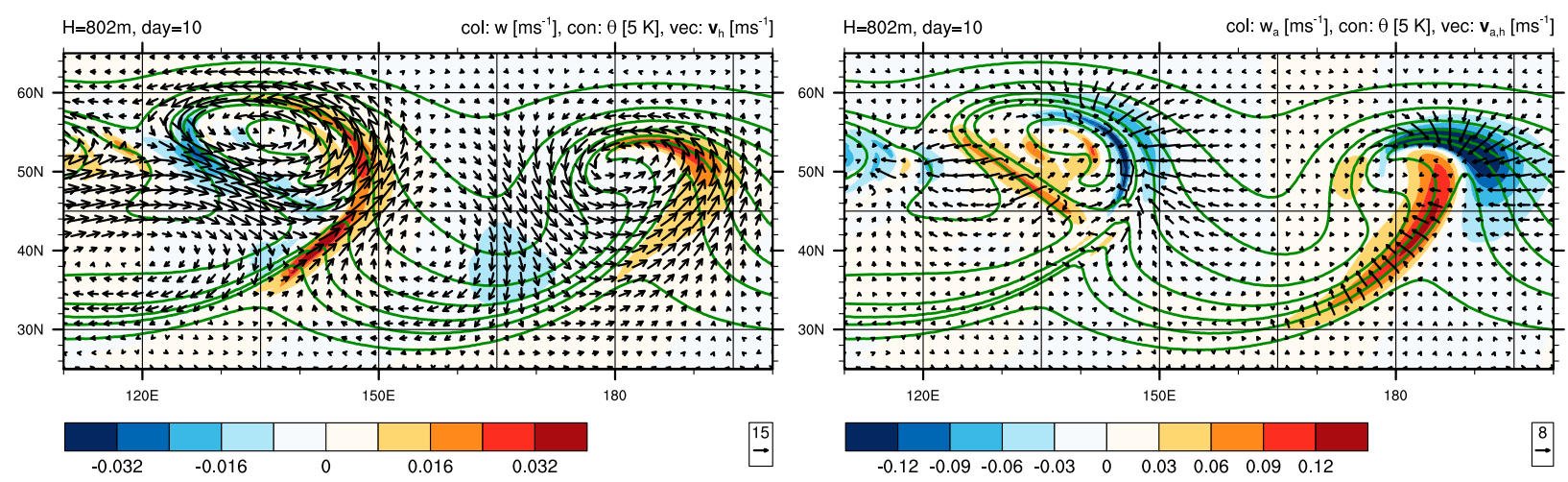

FIG. 2. Potential temperature (green contours) at day 10 at 802-m height. (left) Horizontal wind vectors and vertical wind (colors). (right) Active horizontal wind vectors and vertical active wind (colors). Note the different contour intervals and vector lengths in both panels.

same direction at the left of the rear front of the second cyclone. This signifies a strong along flow acceleration and coincides with the already discussed kinetic energy generation by all possible net forces and net advection. The local tendency of vorticity can already be visually guessed by considering the convergence and divergence of the sum of both $\mathbf{g}$ vectors (bottom panel of Fig. 4) according to (26). Convergence leads to an increase vorticity as is seen all along the east or north of the fronts in Fig. 4. The divergence leads to a decrease of the vorticity and finally anticyclonic flow. Especially at the rear front of the second cyclone this strong divergence is to be found.

\section{c. Vertical structures}

Figure 5 displays vertical slices of kinetic energy and $w, w_{a}$, and $w^{*}$, respectively, along $50^{\circ} \mathrm{N}$. The actual vertical wind pattern (top panel) are associated with enhanced kinetic energy areas, hence jets and fronts. Significant $w_{a}$ and $w^{*}$ amplitudes (middle and bottom panel) are predominantly found in the lower troposphere and especially near the surface. Again, those two fields are similar in shape, but differ in magnitude, $w_{a}$ is stronger than $w^{*}$.

The thermodynamic flow structure, hence the baroclinity, determines the inactive vertical wind $w_{\text {ia }}$. At the surface, the vertical wind vanishes, $w_{s}=0$. Consequently, the active vertical wind must be opposite to the vertical inactive wind there, $w_{a, s}=-w_{\mathrm{ia}, s}$. A inactive flow reaching the surface slantwise from above must spread out at the surface like an Eady edge wave. Hence, a positive vertical active wind must be present at the surface. The bent-back front region at $130^{\circ} \mathrm{E}$ exemplifies this case. When remembering that the inactive wind vector carries also a lot of momentum and kinetic energy, it is clear that the momentum and so the kinetic energy must accumulate near the ground and spread out there. As already discussed, all net kinetic energy conversion terms tend to increase $K$ in the region to the left of this jet. Such a mechanism is known in the literature and has be explored and described under the term sting jet (Slater et al. 2015, 2017). Note, however, that the idealized experiment presented here does not include any boundary layer friction, but only horizontal diffusion.

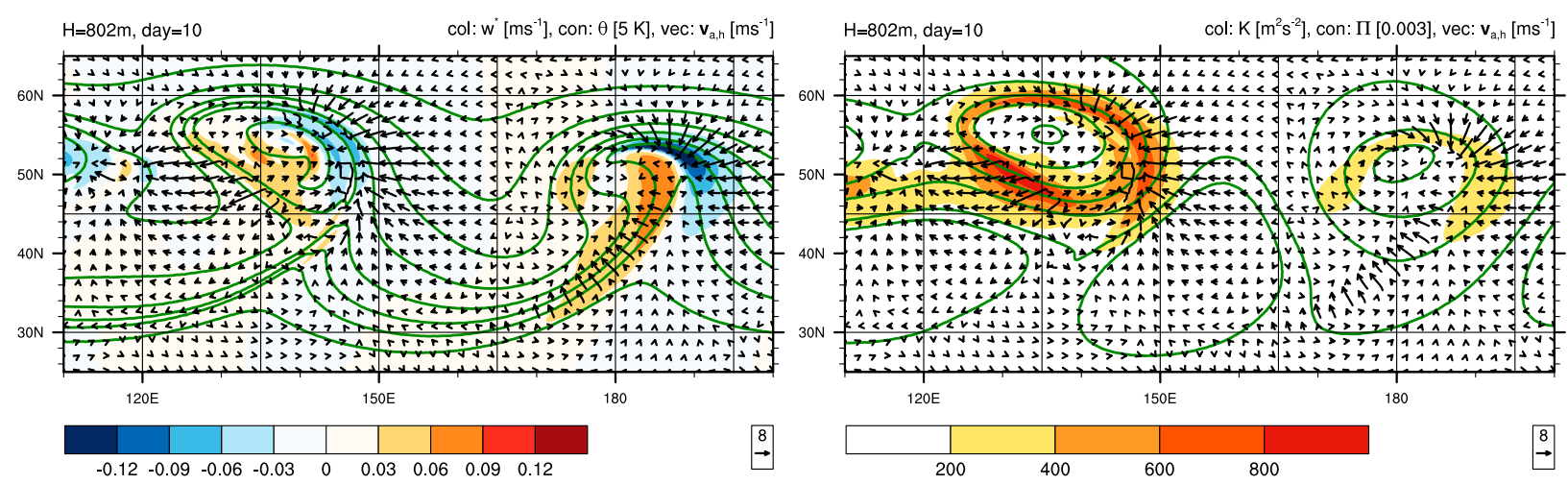

FIG. 3. (left) As in right panel of Fig. 2, but colors show $w^{*}$. (right) Horizontal active wind vectors together with the kinetic energy (colors) and the Exner pressure (green contours) for the same height and time as Fig. 2. 

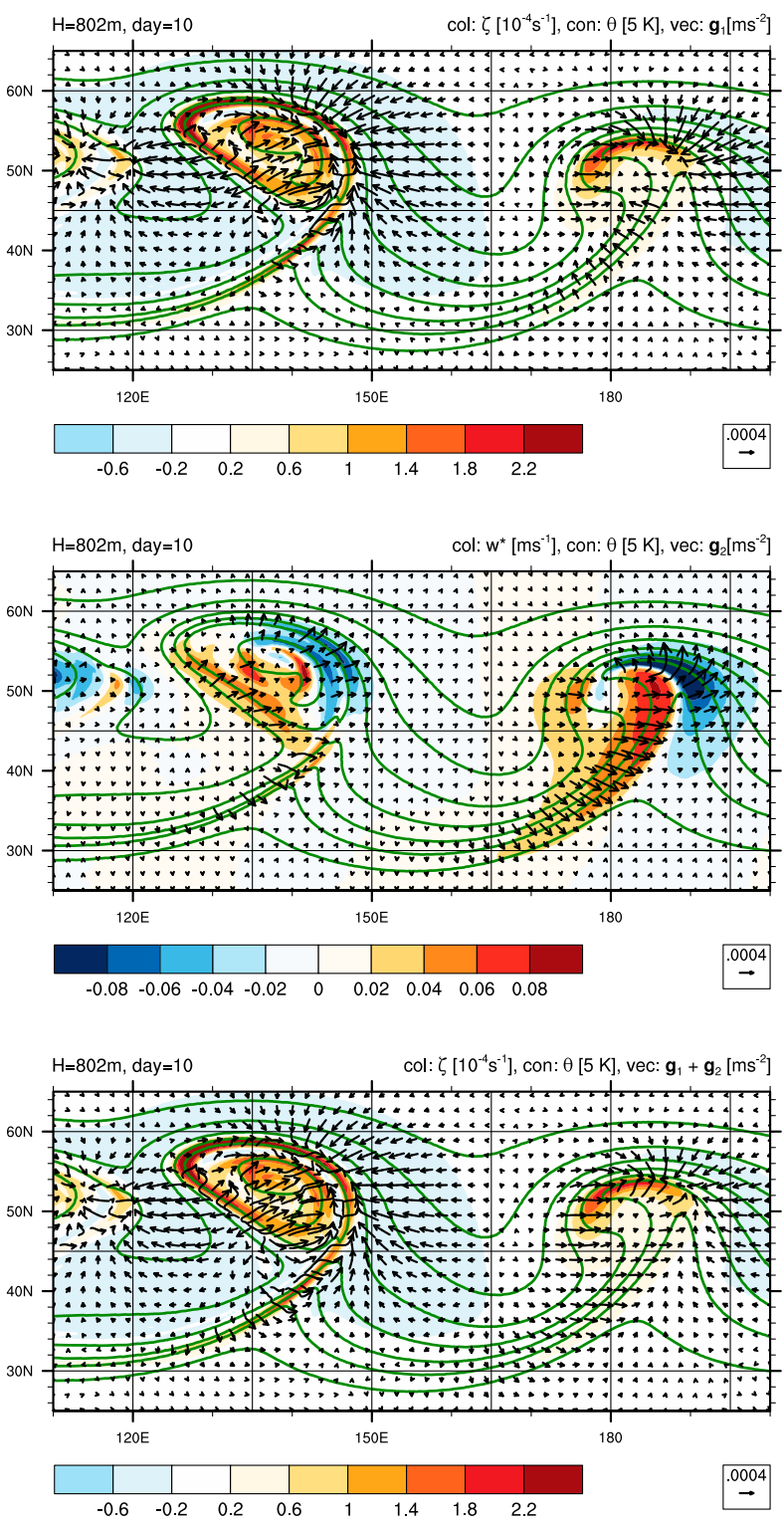

FIG. 4. (top) $\mathbf{g}_{1}$, (middle) $\mathbf{g}_{2}$, and (bottom) $\mathbf{g}_{1}+\mathbf{g}_{2}$ with (top),(bottom) the vorticity $\zeta$ and (middle) the isentropic displacement wind $w^{*}$ in colors at day 10 at $802-\mathrm{m}$ height.

This configuration exaggerates the effect in comparison to the true atmosphere.

The active vertical wind associated with the front at $175^{\circ} \mathrm{W}$ is negative ahead of the front and positive behind the front. This reflects the different signs of baroclinity, hence $w_{\text {ia }}$, on both sides of the front, which is originally due to the initial temperature distribution. The horizontal inactive wind transports heat in different directions, warm air to the north and cold air to the south, which is reflected by the different signs of $w_{\text {ia }}$ according to (11). The inactive flow 'wants' to have an upward component $w_{\text {ia }}$ ahead of the front. This is indeed nearly
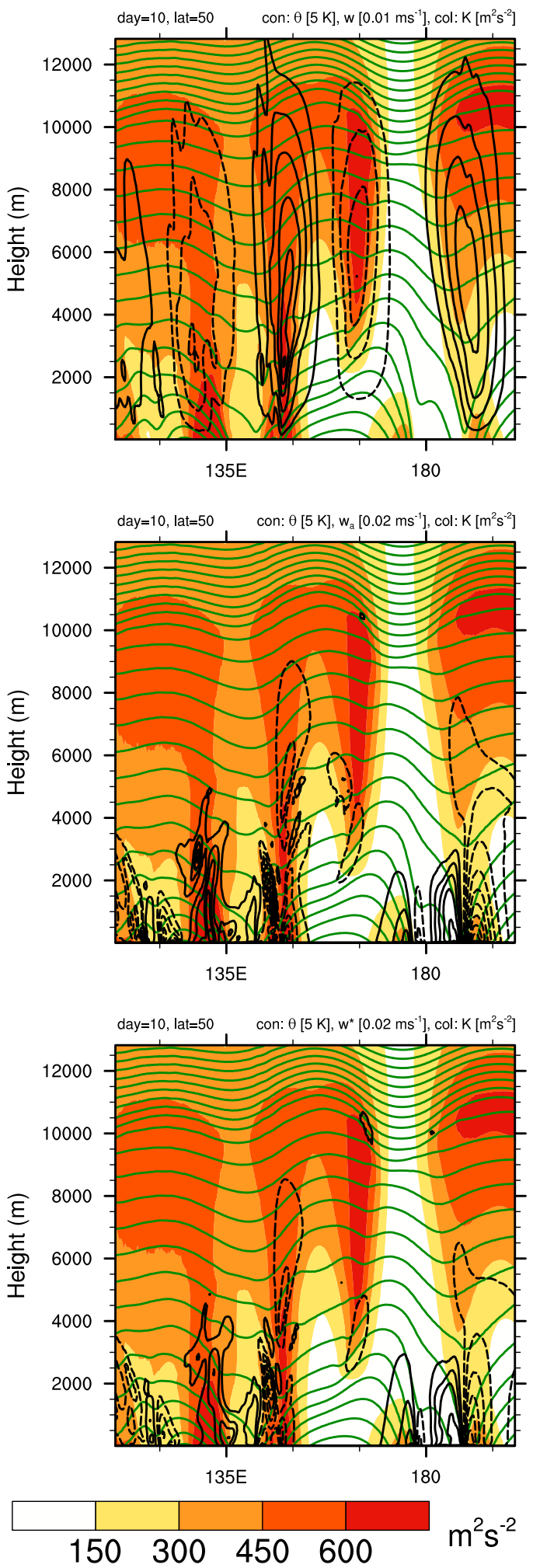

FIG. 5. Vertical slices of kinetic energy (colors) and potential temperature (green contours) along the latitude of $50^{\circ} \mathrm{N}$ at day 10. Black contours are (top) $w$, (middle) $w_{a}$, and (bottom) $w^{*}$. Zero contour lines are not drawn. 

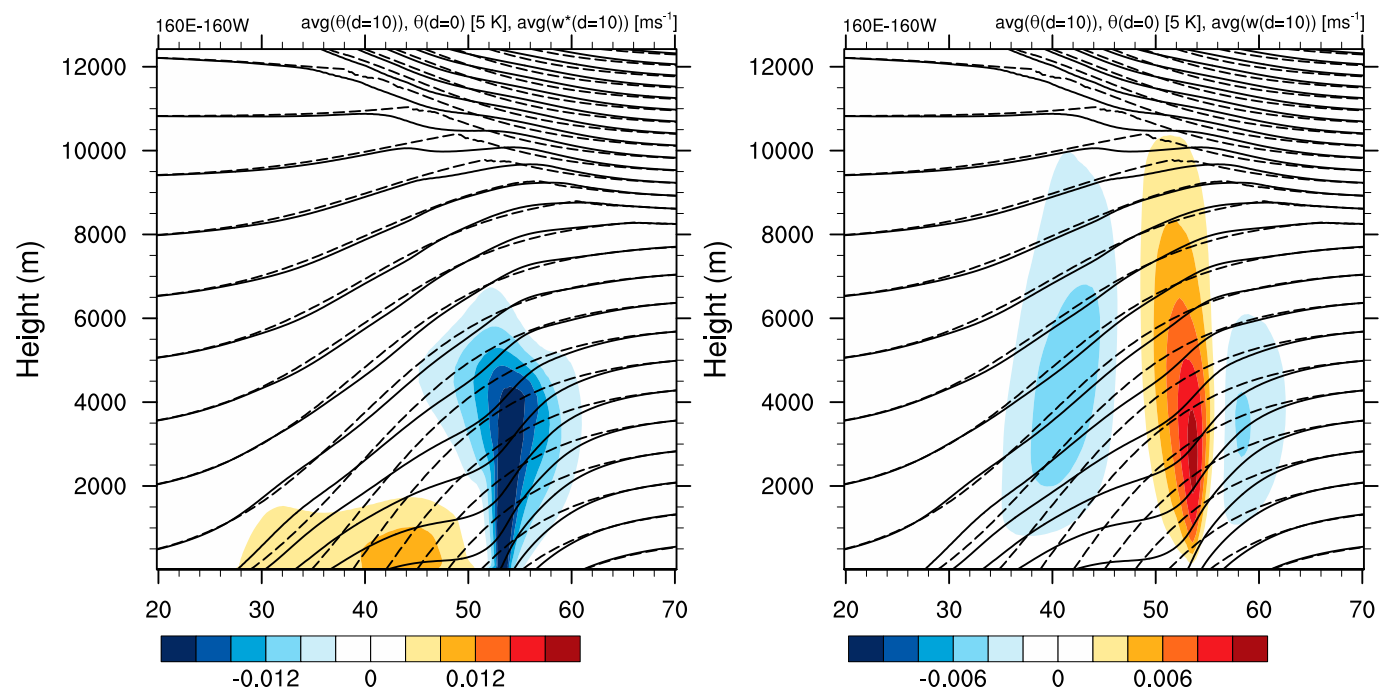

FIG. 6. Zonal sectorial averages between $160^{\circ} \mathrm{E}$ and $160^{\circ} \mathrm{W}$ of (left) $w^{*}$ and (right) $w$ in colors, averages of $\theta$ (solid contours) and initial $\theta$ (dashed contours) at day 10 .

achieved farther up in the midtroposphere, where $w$ becomes positive and $w_{a}$ becomes small. But it is impossible to be achieved near the ground because of the impermeable surface, where $w_{a}$ must then be negative. The inactive flow 'wishes' to be downward behind the front, which is clearly not possible and consequently an upward vertical active wind is present. This positive $w_{a}$ does clearly not reach significantly higher levels.

The pattern of $w^{*}$ around the front at $175^{\circ} \mathrm{W}$ exemplify impressively how isentropes are pushed down ahead of the front and how they are pushed up behind. The isentropic displacement vertical wind changes sign exactly at the local minima of the isentropes. Isentropes shifted downward/upward reflect the adiabatic warming/ cooling in the warm/cold sector of the cyclones. Since cold air is shifted below warm air in a baroclinic wave, upward $w^{*}$ must be found predominantly in lower levels whereas in higher levels, downward $w^{*}$ mirror the warming.

Figure 6 displays the zonally averaged potential temperature profiles for the first cyclone $\left(160^{\circ} \mathrm{E}-160^{\circ} \mathrm{W}\right)$ in comparison to the initial $\theta$ profile. The expected mean flattening of the isentropes is clearly visible. This flattening is achieved by rising and sinking with $w^{*}$ in the lower part of the troposphere (Fig. 6, left). The area where isentropes rise does not reach as high as the sinking area. This averaging of $w^{*}$ indicates just a onecell structure in the troposphere: $w^{*}$ is upward at the equatorward side and downward at the poleward side. If the actual $w$ is averaged (Fig. 6, right), the typical pattern of the Ferrel cell appears with negative values in the subtropical latitudes and positive values in the subpolar latitudes. From this comparison it is concluded that $w^{*}$ can be interpreted as the local brother of the vertical residual wind appearing in the TEM framework.

\section{d. Fronts and gravity wave signatures}

Disentangling vortical motions and gravity waves has always been of great interest in the atmospheric community, especially because the sources of the gravity waves in the troposphere are of interest for the evolution of the flow field higher up in the stratosphere and mesosphere. Conventionally, the full horizontal divergence is invoked for the visual analysis of gravity waves (see e.g., Plougonven and Snyder 2007). However, synopticscale signatures of the divergence associated with the baroclinic wave may dominate the overall pattern. Most frequently, high-pass-filtered divergence or vertical velocity fields are alternatively inspected. In a more sophisticated approach, a balanced velocity field is deduced from a reasonably approximated balance equation. Deviations from this balanced field reflect the gravity wave structures at higher levels (Mirzaei et al. 2017). An alternative approach becomes now possible when considering the transformed divergence equation in (27). Obviously from (27), the rotational part of the $\mathbf{g}$ vectors is the footprint of gravity waves.

Figures 7 and 8 (left) display the rotation of the sum of the $\mathbf{g}$ vectors for different heights and times. Above the lower troposphere the contribution from the $\mathbf{g}_{1}$ vector dominates this sum (not shown). Gravity wave pattern are directly visible in those plots. Large-scale pattern of $\mathbf{k} \cdot \nabla \times \mathbf{g}$ do not dominate the plot, even though they are not fully eliminated as can be seen for the plots in $8-\mathrm{km}$ height. This is expected, because those large-scale 

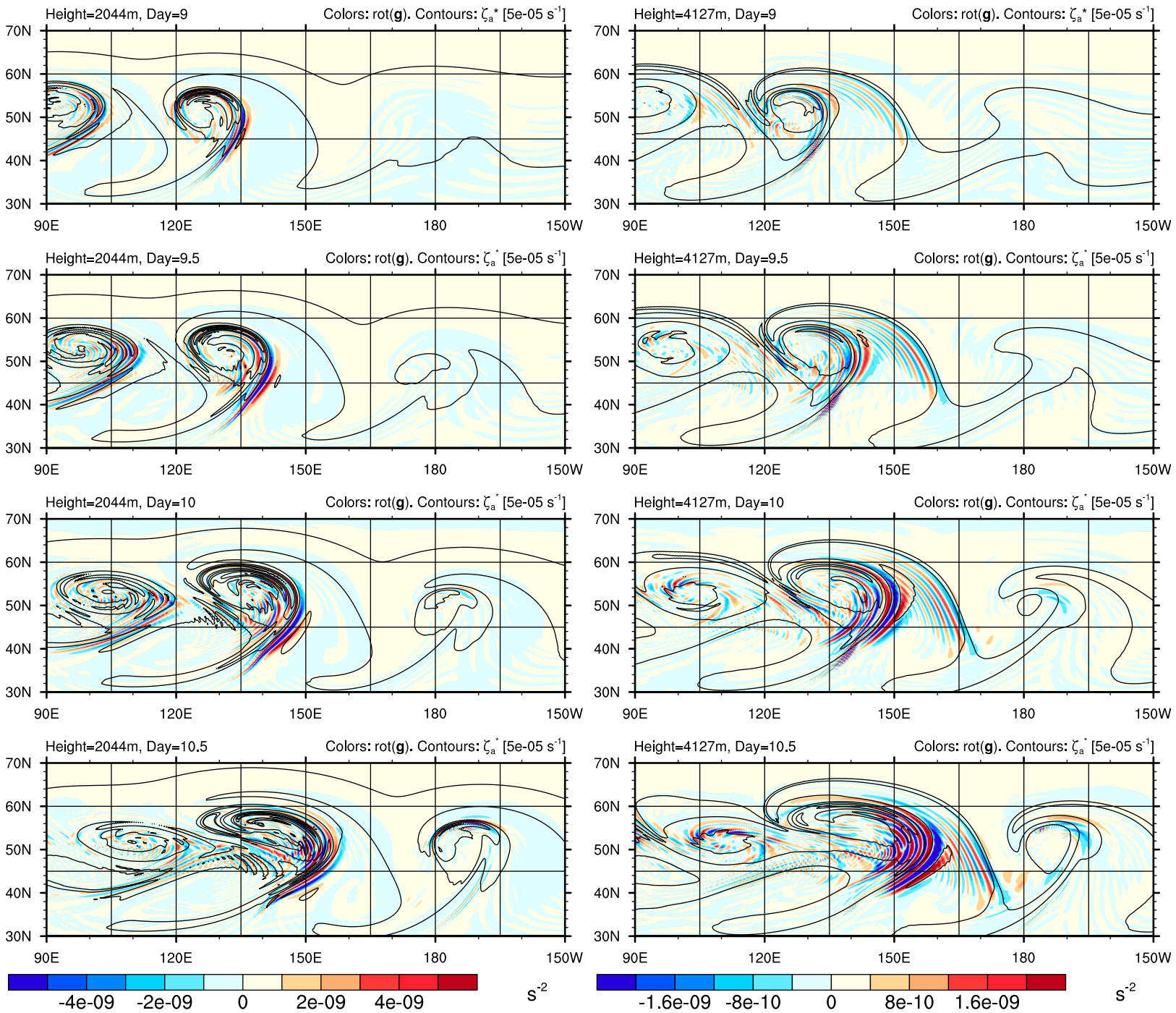

FIG. 7. Forcing to the divergence equation $\mathbf{k} \cdot \nabla \times \mathbf{g}$ (colors) and $\zeta_{a}^{*}$ (contours) for subsequent times and different heights: (left) 2 and (right) $4 \mathrm{~km}$.

patterns reflect the large-scale movement of the full baroclinic wave as it travels toward the East.

The lowermost height of $2 \mathrm{~km}$ (Fig. 7, left) exhibits a lot of waves that develop in coherence with the fronts and the vorticity filaments. Since the model experiment excludes boundary layer friction, those waves are clearly exaggerated compared to a realistic case. For instance, downward traveling waves are reflected at the surface and not dissipated in subgrid-scale turbulence. At all inspected times, the coherence with the $\zeta_{a}^{*}$ filaments is present at 2-km height. The next higher displayed level $(4 \mathrm{~km}$, Fig. 7 , right) reveals that the wave structures of the main front are sometimes no longer exactly parallel to the $\zeta_{a}^{*}$ isolines at day 9 and 10 . This means that those waves where generated below and travel up freely. An upper-level front is visible and coherent wave structures are forming to the west of this front. The back-bent front of the second cyclone is also accompanied by wavy structures parallel to the $\zeta_{a}^{*}$ isolines.

An interesting behavior is to be observed at the height of $8 \mathrm{~km}$ (Fig. 8, left). The wavelengths associated with the upper-level front are notable longer than in 4-km height, they become more pronounced at later times and are not tied to the strongest $\zeta_{a}^{*}$ gradients at $8-\mathrm{km}$ height. This means that this wave must have been traveled from below to this height.

At the height of $12 \mathrm{~km}$ (Fig. 8, right), three main wave packets dominate the picture at days 9, 9.5, and 10 . These are the wave packets of the main front, the backbent front—or sting jet—and the foregoing upper-level front. The wavelength associated with the upper-level jet is again shorter than at $8-\mathrm{km}$ height. 

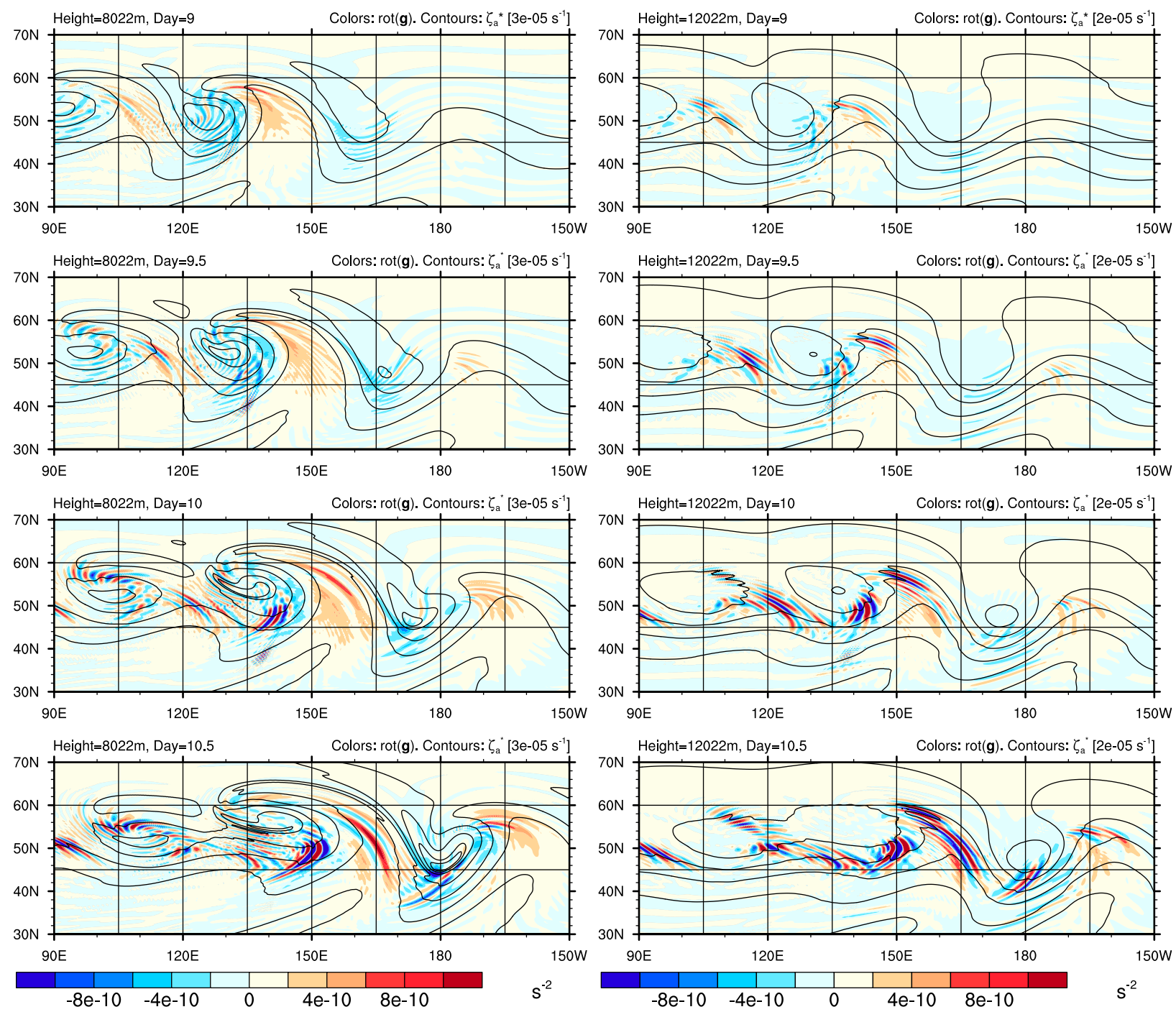

FIG. 8. As in Fig. 7, but for heights of (left) 8 and (right) $12 \mathrm{~km}$.

Figure 9 displays the forcing of the g-vectors to the vorticity equation at day 10 . Intentionally, the color scale is chosen equal to the panels in Figs. 7 and 8. This choice highlights that the forcing to the vorticity is much larger compared to the forcing to the divergence in the troposphere than it is in the stratosphere. In the stratosphere, the forcing is of the same order of magnitude. The gravity wave packets arriving in the stratosphere have a projection onto the vorticity modes, which is not yet so obvious in the troposphere. Vorticity dynamics dominates the whole dynamics in the troposphere. This is expected and confirms the success of quasigeostrophic theory, which filters gravity wave modes, from another perspective.

Three wave packets (Fig. 10) reach the stratosphere and it is of interest to inspect their origin. The wave packet on the left panel of Fig. 10 originates from the lower edge of the elevated front above the sting jet. The front is marked through a strong generation of anticyclonic vorticity (cf. dashed contours). The colored gravity wave pattern originates just along the lower end ( $\approx 2500$-m height) of this front where the wind speed is at a local minimum. Further gravity waves with different properties are tied to the surface fronts, but the ambient flow favors only the discussed wave pattern, the other waves do not propagate away from their origin.

Different wave packets are present in the lower $3 \mathrm{~km}$ in the second cross section (Fig. 10, middle panel). The surface front triggers waves with different properties. Whereas the right wave packet only reaches the midtroposphere, the left wave packet finds an environment that allows for a farther upward propagation. The tilt of the phase lines alters when the wave packet reaches the stratosphere where the static stability increases 

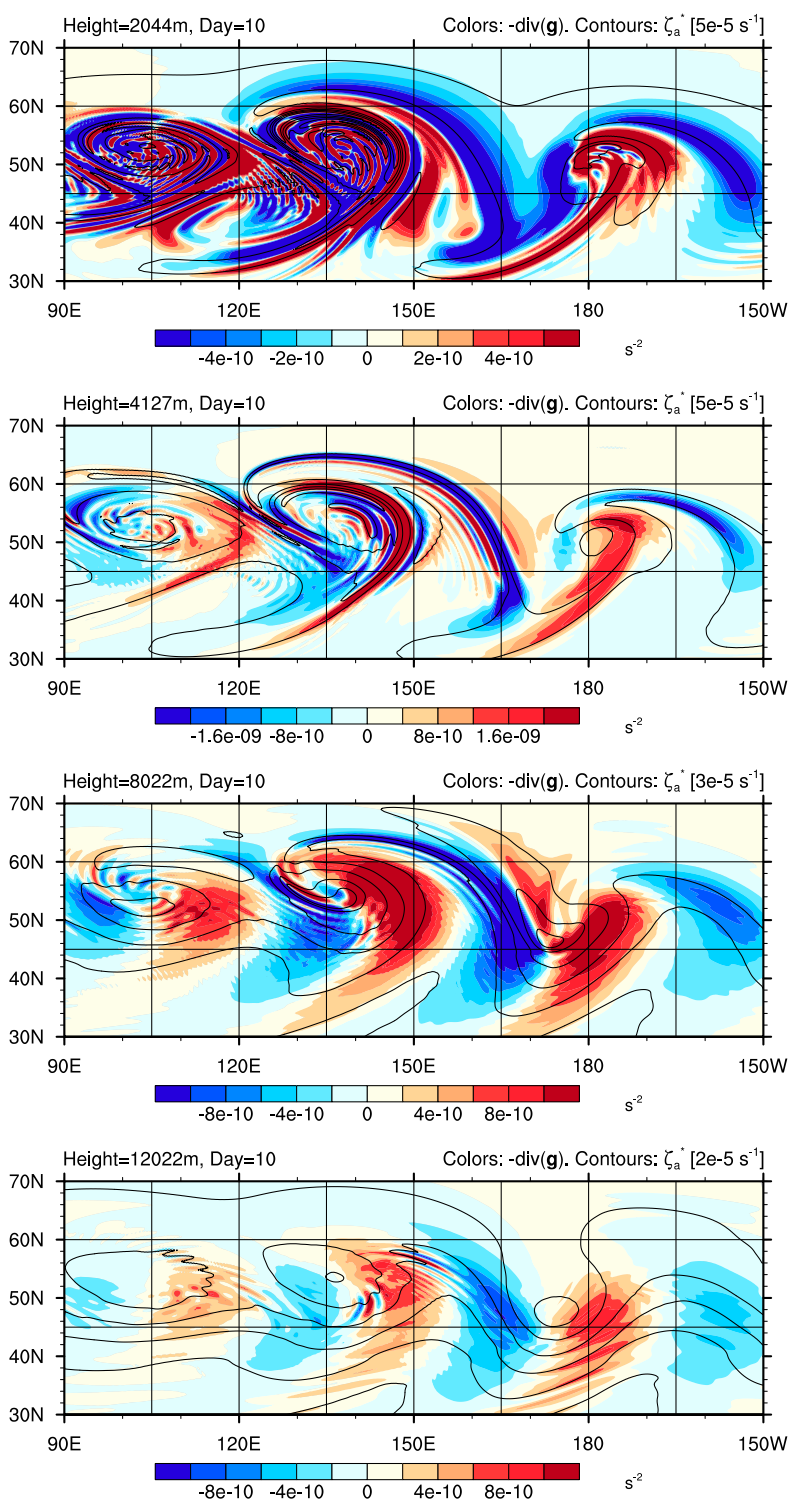

FIG. 9. Forcing to the vorticity equation $-\nabla \cdot \mathbf{g}$ (colors) and $\zeta_{a}^{*}$ (contours) at day 10 for different heights.

and therefore the phase lines become less inclined. In the lower troposphere, the waves from the main front and the back-bent front at the left side of the panel are superposed. This makes it quite difficult to identify unique wave properties.

The third wave packet on the right panel of Fig. 10 is a superposition of a wave generated at the upper-level front in about $5-\mathrm{km}$ height and another wave packet generated at the tropopause level. This upper wave packet has already been described in Plougonven and Snyder (2007) and O'Sullivan and Dunkerton (1995). The vertical wavelength of this wave packet is badly resolved with the chosen vertical level distribution.
The layer thickness is about $400 \mathrm{~m}$ in this height. However, such a level distribution reflects quite well common practice in operational NWP models like ICON (Zängl et al. 2015). The noisy structure seems additionally to arise from the interpolation scheme from the hexagonal mesh to the latitude-longitude grid. The rightmost end of this wave packet is associated with a front in about $10-\mathrm{km}$ height, which is marked by a negative vorticity generation $\left(-\nabla_{h} \cdot \mathbf{g}<0\right)$. At this place, tilted phase lines from upper right to lower left are foreshadowed additionally to the expected phase lines tilted from lower right to upper left. Hence, waves are radiated in both directions, upward and downward. The lower wave packet originating from the front in about $5-\mathrm{km}$ height radiates also in two directions, upward and downward. The similarities between the wave packets excited from the upper-level fronts in the left and the right panel of Fig. 10 are interesting. Both wave packets occur in the lower part of the respective elevated fronts. The fronts are associated with the generation of anticyclonic shear (dashed lines) that comes along with a small patch of positive vorticity generation (solid lines) just upstream of it. This is in line with previous knowledge that the excitation of waves is associated with strong frontogenesis (Charron and Manzini 2002).

The described wave packets are typically to be found in other simulations, like in O'Sullivan and Dunkerton (1995), Plougonven and Zhang (2014), Kim et al. (2016), or Mirzaei et al. (2017). Those authors identified surface fronts or upper-level jet streaks (upper-level fronts) with the excitation of gravity waves. As the sharpness of the fronts depends on model resolution, the modeled physical properties of the waves also depend on model resolution.

\section{Conclusions}

A definition of the three-dimensional inactive state of the atmosphere has been given. The associated EPV flux $\nabla \theta \times \nabla B$ is nondivergent and therefore does not contribute to a tendency in the EPV budget equation. The part of the flow representing the inactive state has been made invisible in the prognostic equations. The resulting transformed equations reveal that the dynamics for vortical modes as well as for gravity wave modes depend only on the horizontal active vorticity flux and vertical advection with $w^{*}$. The $\theta$ equation is then characterized by the vertical movement of the isentropes by the isentropic displacement vertical wind $w^{*}$. This new diagnostic viewpoint does not introduce new physics or new insights into physical processes, but helps to extract significant terms and distinguishes them from the insignificant terms that cancel out each other. Furthermore, it brings aspects of previously averaged dynamics, as 

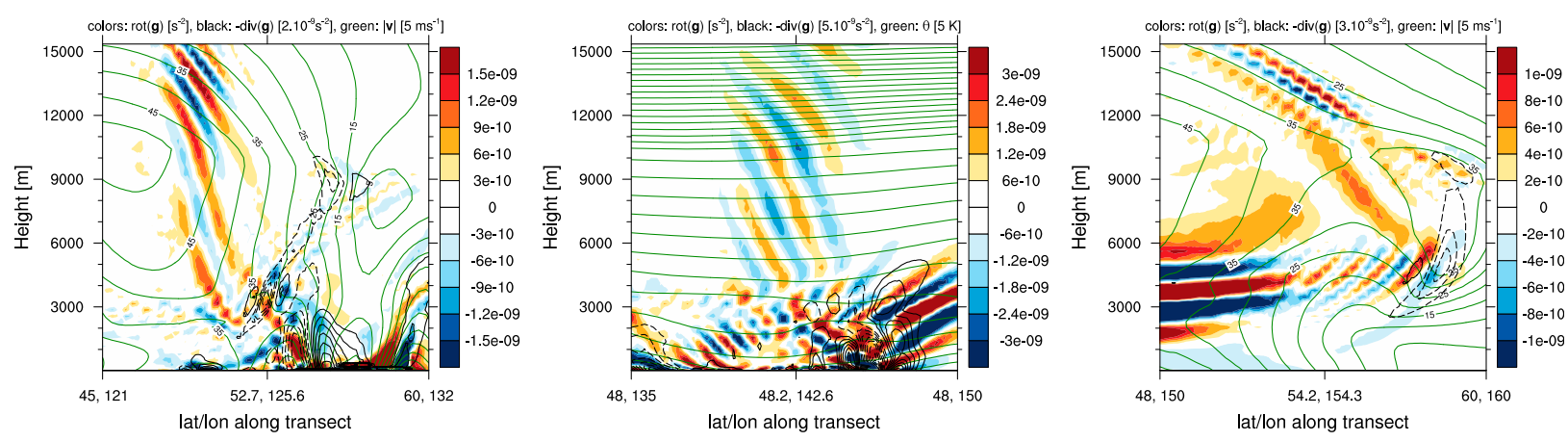

FIG. 10. Transects of the three wave packets reaching the stratosphere: (left) the sting jet (bent-back front) wave packet, (middle) the surface front wave packet, and (right) the upper-level front wave packets. The rotation of $\mathbf{g}$ is given in colors and its negative divergence is given as black contours. Green lines are (middle) $\theta$ and (left),(right) $|\mathbf{v}|$.

seen in the TEM equations, closer to local dynamics and serves thus to find relations between different viewpoints.

The first special aspect of the work is the discussion of the vertical inactive and active winds. Because the inactive wind blows along sloped isentropes, it has a vertical component. Isentropes may intersect the ground and therefore the inactive vertical wind cannot coincide with the actual vertical wind near the surface. Consequently, a deviation from the inactive vertical wind, the active vertical wind, must be present and determine the dynamics. The vertical active wind is quite similar to the isentropic displacement vertical wind in shape. This allows for an easy analysis of the thermal structures of baroclinic waves or other dynamic events that lead to vertical displacements of isentropic surfaces as for instance during stratospheric warmings. Isentropes may alternatively also change their heights by diabatic processes. The competing effects of those dynamic and diabatic effects shape the mean structure of isentropes in the atmosphere [see Papritz and Spengler (2015)].

The second special aspect is that the distinction between gravity wave modes and vortical modes lies only in the properties of the active vorticity flux $\mathbf{g}=\mathbf{g}_{1}+\mathbf{g}_{2}=$ $\mathbf{v}_{a, h} \zeta_{a}^{*}-w^{*} \boldsymbol{\omega}_{h}$. The divergent part of this vector determines vortical modes and the rotational part is responsible for gravity wave modes. The diagnosis of the rotational part of the vorticity flux gives insight into the generation, propagation and breaking of gravity waves.

As a future work, the contribution of the forcing terms in the transformed momentum equation will be inspected with regard to the generation of the kinetic energy cascade. Spectral fluxes due to vortical modes and divergent modes can be separated in such an analysis. Large opposing spectral fluxes due to different tendencies will be avoided, because the inactive terms cancel out beforehand. This already envisioned future work will give insights into the different shapes of the kinetic energy spectrum in different heights of the atmosphere.

Acknowledgments. This paper is a contribution to the project T1 (Mesoscale energy cascades in the lower and middle atmosphere) of the Collaborative Research Centre TRR 181 "Energy Transfer in Atmosphere and Ocean" funded by the Deutsche Forschungsgemeinschaft (DFG, German Research Foundation) Projektnummer 274762653.

\section{APPENDIX A}

\section{Initial Conditions for Baroclinic Wave Test Case}

The initial zonal jet obeys the thermal wind balance, hence

$$
-\partial_{z} u+\frac{N^{2}}{g} u=\frac{g}{f a} \partial_{\varphi} \ln \theta
$$

Constant values are chosen as surface boundary conditions for wind and Exner pressure: $u_{\text {surf }}=0$ and $\Pi_{\text {surf }}=1$. The static stability in the stratosphere is set constant: $N_{\text {strat }}^{2}=5 \times 10^{-4} \mathrm{~s}^{-2}$. The tropopause height $H_{\text {pause, }}$, the surface potential temperature $\theta_{\text {surf }}$, and the static stability in the troposphere $N_{\text {trop }}^{2}$ are represented by the following generic function

$$
\begin{gathered}
\eta(\varphi)=\alpha+\beta\{\tan h[\gamma(\varphi+\delta)]+\tan h[\gamma(-\varphi+\delta)]\} \\
\partial_{\varphi} \eta(\varphi)=\beta \gamma\left\{-\tan h^{2}[\gamma(\varphi+\delta)]+\tan ^{2}[\gamma(-\varphi+\delta)]\right\},
\end{gathered}
$$

where the parameters $\alpha, \beta, \gamma$, and $\delta$ can be chosen freely. The specific choices are summarized in Table A1. 
TABLE A1. Summary of initial condition parameters.

\begin{tabular}{lcccc}
\hline \multicolumn{1}{c}{$\eta(\varphi)$} & $\alpha$ & $\beta$ & $\gamma$ & $\delta$ \\
\hline$H_{\text {pause }}(\mathrm{km})$ & 8 & 2.5 & 3.9 & $0.27 \pi$ \\
$\theta_{\text {surf }}(\mathrm{K})$ & 250 & 25 & 3.9 & $0.25 \pi$ \\
$N_{\text {trop }}^{2}\left(10^{-4} \mathrm{~s}^{-2}\right)$ & 2.5 & -0.75 & 3.9 & $0.25 \pi$ \\
\hline
\end{tabular}

The potential temperatures in the troposphere and in the stratosphere are then

$$
\begin{aligned}
& \theta_{\text {trop }}(\varphi, z)=\theta_{\text {surf }}(\varphi) e^{N_{\text {trop }}^{2}(\varphi) z / g}, \\
& \theta_{\text {strat }}(\varphi, z)=\theta_{\text {surf }}(\varphi) e^{H_{\text {pause }}(\varphi)\left[N_{\text {trop }}^{2}(\varphi)-N_{\text {strat }}^{2}\right] / g+N_{\text {strat }}^{2} / g} .
\end{aligned}
$$

Logarithmic meridional derivatives are needed for the thermal wind balance (A1)

$$
\begin{aligned}
\partial_{\varphi} \ln \theta_{\text {trop }}(\varphi, z)= & \frac{\partial_{\varphi} \theta_{\text {surf }}(\varphi)}{\theta_{\text {surf }}(\varphi)}+\frac{z}{g} \partial_{\varphi} N_{\text {trop }}^{2}(\varphi), \quad \text { (A6) } \\
\partial_{\varphi} \ln \theta_{\text {strat }}(\varphi)= & \frac{\partial_{\varphi} \theta_{\text {surf }}(\varphi)}{\theta_{\text {surf }}(\varphi)}+\partial_{\varphi} H_{\text {pause }}(\varphi) \frac{N_{\text {trop }}^{2}(\varphi)-N_{\text {strat }}^{2}}{g} \\
& +\partial_{\varphi} N_{\text {trop }}^{2}(\varphi) \frac{H_{\text {pause }}(\varphi)}{g} .
\end{aligned}
$$

The solution of the nonhomogeneous differential equation in (A1) differs again in the stratosphere and in the troposphere. For the troposphere, the solution is

$$
u(\varphi, z)=\left(\frac{\tau_{1}}{\tau_{3}}+\frac{\tau_{2}}{\tau_{3}^{2}}\right)\left(1-e^{\tau_{3} z}\right)+\frac{\tau_{2}}{\tau_{3}} z,
$$

where the abbreviations $\tau_{i}$ are

$$
\tau_{1}=\frac{g}{a f(\varphi)} \frac{\partial_{\varphi} \theta_{\text {surf }}(\varphi)}{\theta_{\text {surf }}(\varphi)}, \quad \tau_{2}=\frac{\partial_{\varphi} N_{\text {trop }}^{2}(\varphi)}{a f(\varphi)}, \quad \tau_{3}=\frac{N_{\text {trop }}^{2}(\varphi)}{g} .
$$

The wind in the stratosphere is determined via

$$
\begin{aligned}
u(\varphi, z)= & \left\{\left(\frac{\tau_{1}}{\tau_{3}}+\frac{\tau_{2}}{\tau_{3}^{2}}\right)\left[1-e^{\tau_{3} H_{\text {pause }}(\varphi)}\right]+\frac{\tau_{2}}{\tau_{3}} H_{\text {pause }}(\varphi)\right\} \\
& \times e^{\sigma_{3}\left[z-H_{\text {pause }}(\varphi)\right]}+\frac{\sigma_{1}}{\sigma_{3}}\left\{1-e^{\sigma_{3}\left[z-H_{\text {pause }}(\varphi)\right]}\right\} .
\end{aligned}
$$

The abbreviations $\sigma_{i}$ are

$$
\sigma_{1}=\frac{g}{a f(\varphi)} \partial_{\varphi} \ln \theta_{\text {strat }}(\varphi), \quad \sigma_{3}=\frac{N_{\text {strat }}^{2}}{g}
$$

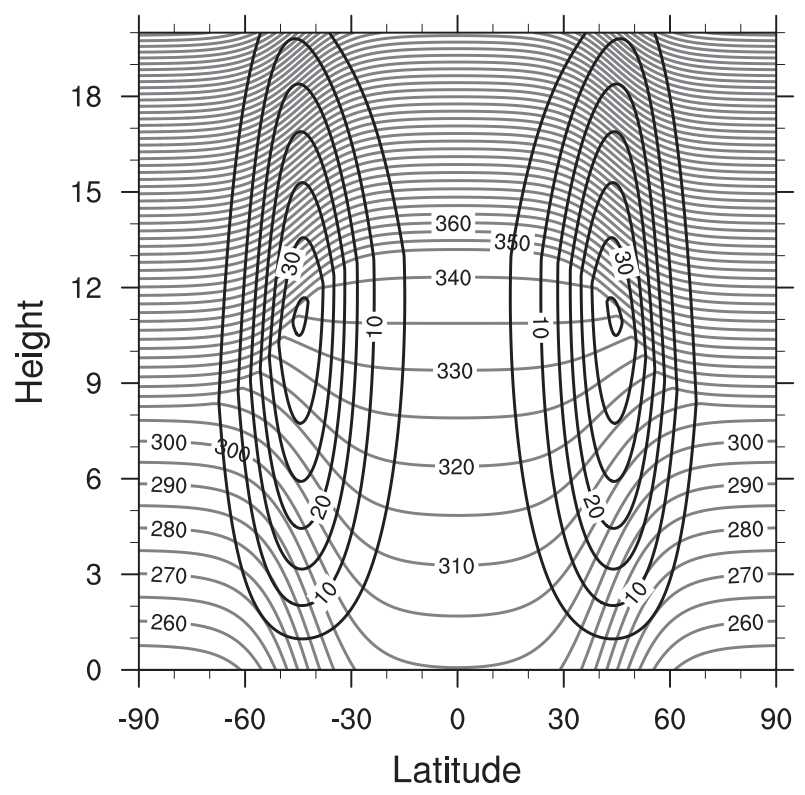

FIG. A1. Initial conditions for $u\left(\mathrm{~m} \mathrm{~s}^{-1}\right)$ (black) and $\theta(\mathrm{K})$ (gray) for the baroclinic wave.

The pressure is determined by a discrete integration of the hydrostatic balance.

Initial conditions for wind and potential temperature are displayed in Fig. A1.

The triggering of the baroclinic wave is accomplished by a negative perturbation on the potential temperature, which is maximum with $-1 \mathrm{~K}$ at the surface and has a height decay according to $\exp \left[-(h / 5000 \mathrm{~m})^{2}\right]$. The horizontal distribution of the perturbation is adapted from Jablonowski and Williamson (2006).

\section{APPENDIX B}

\section{Inactive Flow in Two Dimensions}

It might appear that the concept of inactive flow does not work in two dimensions, as for instance a vertical slice in the $x-z$ plane. It is now explained how it works nevertheless. In that case, the $y$ direction is irrelevant and all terms in the $v$ equation are identically zero:

$$
\partial_{\underbrace{v}_{=0}}^{v}=-\underbrace{\omega_{z}}_{=0} u+\underbrace{\omega_{x}}_{=0} w-\underbrace{\partial_{y} K}_{=0}-c_{p} \theta \underbrace{\partial_{y} \Pi}_{=0}+\underbrace{F_{r, y}}_{=0} .
$$

However, this does not mean that $\partial_{y} \theta$ vanishes. This gradient can be considered as arbitrarily nonzero, $\partial_{y} \theta \neq$ 0 . Therefore, the pressure gradient term can be written as $-c_{p} \theta \partial_{y} \Pi=-c_{p} \partial_{y}(\Pi \theta)+c_{p} \Pi \partial_{y} \theta=0$, where both addends become significant for themselves. With that in 
mind, all computations can be done as in the threedimensional case, and $\partial_{y} \theta$ is considered as significant. Then, $\zeta_{a}^{*}=\omega_{y}\left(\partial_{y} \theta / \partial_{z} \theta\right)$, and

$$
\begin{aligned}
u_{\mathrm{ia}} & =\left(\partial_{y} \theta / \partial_{z} \theta\right)\left(\partial_{z} K / \zeta_{a}^{*}\right)=\partial_{z} K / \omega_{y}, \\
w_{\mathrm{ia}} & =-\left(\partial_{y} \theta / \partial_{z} \theta\right)\left(c_{p} \theta \partial_{x} \Pi+\partial_{x} K\right) / \zeta_{a}^{*} \\
& =-\left(c_{p} \theta \partial_{x} \Pi+\partial_{x} K\right) / \omega_{y} .
\end{aligned}
$$

As long as the horizontal vorticity $\omega_{y}=\partial_{z} u-\partial_{x} w$ does not vanish, the inactive flow can be determined also in the two-dimensional case.

\section{REFERENCES}

Andrews, D. G., and M. E. McIntyre, 1976: Planetary waves in horizontal and vertical shear: The generalized Eliassen-Palm relation and the mean zonal acceleration. J. Atmos. Sci., 33, 2031-2048, https://doi.org/10.1175/1520-0469(1976)033<2031: PWIHAV $>2.0 . \mathrm{CO} ; 2$.

— J. Holton, and C. Leovy, 1987: Middle Atmosphere Dynamics. International Geophysics Series, Vol. 40, Academic Press, 489 pp.

Bannon, P. R., J. Schmidli, and C. Schär, 2003: On potential vorticity flux vectors. J. Atmos. Sci., 60, 2917-2921, https://doi.org/ 10.1175/1520-0469(2003)060<2917:OPVFV > 2.0.CO;2.

Boyd, J. P., 1976: The noninteraction of waves with the zonally averaged flow on a spherical Earth and the interrelationships of eddy fluxes of energy, heat, and momentum. J. Atmos. Sci., 33, 2285-2291, https://doi.org/10.1175/1520-0469(1976)033<2285: TNOWWT $>2.0$.CO;2.

Charron, M., and E. Manzini, 2002: Gravity waves from fronts: Parameterization and middle atmosphere response in a general circulation model. J. Atmos. Sci., 59, 923-941, https://doi.org/ 10.1175/1520-0469(2002)059<0923:GWFFPA>2.0.CO;2.

Davies-Jones, R., 2003: Comments on “A generalization of Bernoulli's Theorem.” J. Atmos. Sci., 60, 2039-2041, https://doi.org/10.1175/ 1520-0469(2003)060<2039:COAGOB > 2.0.CO;2.

Egger, J., K.-P. Hoinka, and T. Spengler, 2015: Aspects of potential vorticity fluxes: Climatology and impermeability. J. Atmos. Sci., 72, 3257-3267, https://doi.org/10.1175/JAS-D-14-0196.1.

Gassmann, A., 2013: A global hexagonal C-grid non-hydrostatic dynamical core (ICON-IAP) designed for energetic consistency. Quart. J. Roy. Meteor. Soc., 139, 152-175, https://doi.org/10.1002/ qj. 1960.

- 2014: Deviations form a general nonlinear wind balance: Local and zonal-mean perspectives. Meteor. Z., 23, 467-481, https://doi.org/10.1127/metz/2014/0568.

- 2018: Discretization of generalized Coriolis and friction terms on the deformed hexagonal C-grid. Quart. J. Roy. Meteor. Soc., 144, 2038-2053, https://doi.org/10.1002/qj.3294.

Haynes, P. H., and M. E. McIntyre, 1990: On the conservation and impermeability theorems for potential vorticity. J. Atmos. Sci., 47, 2021-2031, https://doi.org/10.1175/1520-0469(1990)047<2021: OTCAIT $>2.0 . C O ; 2$.

Hoskins, B., M. Pedder, and D. W. Jones, 2003: The omega equation and potential vorticity. Quart. J. Roy. Meteor. Soc., 129, 3277-3303, https://doi.org/10.1256/qj.02.135.

Jablonowski, C., and D. L. Williamson, 2006: A baroclinic instability test case for atmospheric model dynamical cores. Quart. J. Roy. Meteor. Soc., 132, 2943-2975, https://doi.org/10.1256/qj.06.12.
Kim, Y.-H., H.-Y. Chun, S.-H. Park, I.-S. Song, and H.-J. Choi, 2016: Characteristics of gravity waves generated in the jet-front system in a baroclinic instability simulation. Atmos. Chem. Phys., 16, 4799-4815, https://doi.org/10.5194/acp-16-4799-2016.

Lange, H.-J., 2002: Die Physik des Wetters und des Klimas (The Physics of Weather and Climate). Dietrich Reimer Verlag Berlin, 625 pp.

Mirzaei, M., A. R. Mohebalhojeh, C. Zülicke, and R. Plougonven, 2017: On the quantification of imbalance and inertia-gravity waves generated in numerical simulations of moist baroclinic waves using the WRF model. J. Atmos. Sci., 74, 4241-4263, https://doi.org/10.1175/JAS-D-16-0366.1.

Müller, A., P. Névir, and R. Klein, 2018: Scale dependent analytical investigation of the dynamic state index concerning the quasigeostrophic theory. Math. Climate Wea. Forecasting, 4, 1-22, https://doi.org/10.1515/mcwf-2018-0001.

Névir, P., 2004: Ertel's vorticity theorems, the particle relabelling symmetry and the energy-vorticity theory of fluid mechanics. Meteor. Z., 13, 485-498, https://doi.org/10.1127/0941-2948/ 2004/0013-0485.

O'Sullivan, D., and T. J. Dunkerton, 1995: Generation of inertiagravity waves in a simulated life cycle of baroclinic instability. J. Atmos. Sci., 52, 3695-3716, https://doi.org/10.1175/15200469(1995)052<3695:GOIWIA > 2.0.CO;2.

Papritz, L., and T. Spengler, 2015: Analysis of the slope of isentropic surfaces and its tendencies over the North Atlantic. Quart. J. Roy. Meteor. Soc., 141, 3226-3238, https://doi.org/ 10.1002/qj.2605.

Phillips, N. A., 1963: Geostrophic motion. Rev. Geophys., 1, 123176, https://doi.org/10.1029/RG001i002p00123.

Plougonven, R., and C. Snyder, 2007: Inertia-gravity waves spontaneously generated by jets and fronts. Part I: Different baroclinic life cycles. J. Atmos. Sci., 64, 2502-2520, https://doi.org/ 10.1175/JAS3953.1.

_- and F. Zhang, 2014: Internal gravity waves from atmospheric jets and fronts. Rev. Geophys., 52, 33-76, https://doi.org/10.1002/ 2012RG000419.

Raymond, D. J., and H. Jiang, 1990: A theory for long-lived mesoscale convective systems. J. Atmos. Sci., 47, 3067-3077, https://doi.org/ 10.1175/1520-0469(1990)047<3067:ATFLLM>2.0.CO;2.

Sadourny, R., 1975: The dynamics of finite-difference models of the shallow-water equations. J. Atmos. Sci., 32, 680-689, https://doi.org/ 10.1175/1520-0469(1975)032<0680:TDOFDM >2.0.CO;2.

Schär, C., 1993: A generalization of Bernoulli's theorem. J. Atmos. Sci., 50, 1437-1443, https://doi.org/10.1175/1520-0469(1993) $050<1437$ :AGOBT $>2.0 . \mathrm{CO} ; 2$.

- 2003: Reply. J. Atmos. Sci., 60, 2042-2043, https://doi.org/ 10.1175/1520-0469(2003)060<2042:R > 2.0.CO;2.

Slater, T. P., D. M. Schultz, and G. Vaughan, 2015: Acceleration of near-surface strong winds in a dry, idealised extratropical cyclone. Quart. J. Roy. Meteor. Soc., 141, 1004-1016, https:// doi.org/10.1002/qj.2417.

,-- , and ——, 2017: Near-surface strong winds in a marine extratropical cyclone: acceleration of the winds and the importance of surface fluxes. Quart. J. Roy. Meteor. Soc., 143 , 321-332, https://doi.org/10.1002/qj.2924.

Weber, T., and P. Névir, 2008: Storm tracks and cyclone development using the theoretical concept of the Dynamic State Index (DSI). Tellus, 60A, 1-10, https://doi.org/10.1111/j.1600-0870.2007.00272.x.

Zängl, G., D. Reinert, P. Ripodas, and M. Baldauf, 2015: The ICON (ICOsahedral Non-hydrostatic) modelling framework of DWD and MPI-M: Description of the non-hydrostatic dynamical core. Quart.J. Roy. Meteor. Soc., 141, 563-579, https:// doi.org/10.1002/qj.2378. 